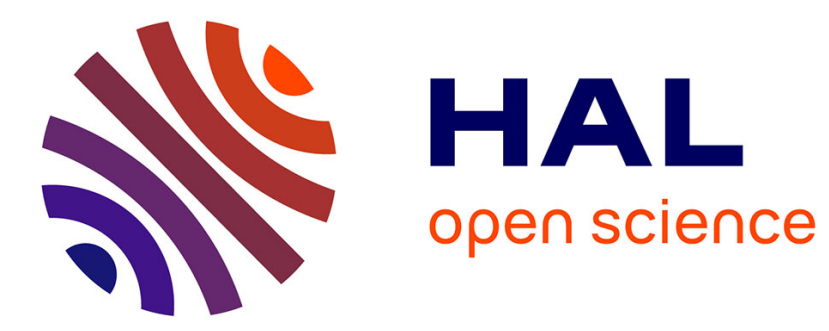

\title{
Sensorium Dei. Espace et présence sensible de l'esprit chez Newton \\ Philippe Hamou
}

\section{To cite this version:}

Philippe Hamou. Sensorium Dei. Espace et présence sensible de l'esprit chez Newton. Revue philosophique de la France et de l'étranger, 2014, 139 (1), pp.47-72. 10.3917/rphi.141.0047 . hal01551263

\section{HAL Id: hal-01551263 \\ https://hal.parisnanterre.fr/hal-01551263}

Submitted on 22 Oct 2019

HAL is a multi-disciplinary open access archive for the deposit and dissemination of scientific research documents, whether they are published or not. The documents may come from teaching and research institutions in France or abroad, or from public or private research centers.
L'archive ouverte pluridisciplinaire $\mathbf{H A L}$, est destinée au dépôt et à la diffusion de documents scientifiques de niveau recherche, publiés ou non, émanant des établissements d'enseignement et de recherche français ou étrangers, des laboratoires publics ou privés. 


\section{SENSORIUM DEI \\ ESPACE ET PRÉSENCE SENSIBLE DE L'ESPRIT CHEZ NEWTON}

Je me propose d'offrir ici ${ }^{1}$ quelques éléments de contexte et quelques pistes interprétatives pour l'élucidation de la fameuse définition newtonienne de l'espace qu'on trouve dans les Questions de l'Optique, à partir de l'édition latine de $1706^{2}$. L'espace y est dit être le sensorium dei, ou, plus prudemment, tanquam sensorium dei : comme, ou pour ainsi dire, le sensorium de Dieu - le lieu dans lequel Dieu perçoit toute chose par sa présence immédiate, et dans lequel il forme et réforme les parties de l'univers, tout comme l'homme lui-même perçoit les images des choses dans un certain espace de son cerveau (son sensorium), et y agit sur les esprits animaux qui contrôlent le mouvement des muscles. La métaphore a certainement une intention pédagogique. Newton veut donner à entendre quelque chose au sujet de la présence de Dieu au monde, et il le fait par le biais d'un modèle humain (ou animal). Contrairement à ce que Leibniz voudra lui imputer, il ne s'agit pas de ravaler Dieu au rang des créatures, ni d'élever l'espace au rang de la divinité, mais plutôt, dans un régime de discours analogique

1. Ce texte a été rédigé dans le cadre d'un projet de recherche soutenu par l'Agence nationale de la Recherche (programme PNEUMA, "L'espace de l'esprit », ANR-09-SSOC-056).

2. Il s'agit de la traduction latine (par Samuel Clarke) de l'Opticks de 1704 (I. Newton, Optice: sive de reflexionibus, refractionibus, inflexionibus \& coloribus lucis libri tres, Londini, 1706 ; édition numérique www.newtonproject.sussex. ac.uk, site consulté en février 2013). Newton y amplifie très largement le texte des Queries conclusives du Livre III. Le principal texte sur le sensorium se trouve dans la Quaestio 20 : «Annon ex phænomenis constat, esse Entem Incorporeum, Viventem, Intelligentem, Omnipræsentem, qui in Spatio infinito, tanquam Sensorio suo, res Ipsas intime cernat, penitusque perspiciat, totasque intra se præsens præsentes complectatur; quarum quidem rerum Id quod in nobis sentit \& cogitat, Imagines tantum ad se per Organa Sensuum delatas, in Sensoriolo suo percipit \& contuetur. » Voir infra, page 56 et note 23 , la traduction française de ce passage.

Revue philosophique, $\mathrm{n}^{\circ} 1 / 2014$, p. 47 à p. 72 
que Newton identifie explicitement, d'accommoder notre langage sur Dieu aux pouvoirs de la compréhension humaine ${ }^{3}$. Selon le postulat tacite, sous-jacent à cet usage pédagogique de la métaphore, la présence de l'âme humaine dans son sensorium, l'action qu'elle y exerce sur les images cérébrales et sur les esprits animaux nous sont suffisamment connues pour rendre intelligible, au moins analogiquement, le mode de présence et l'action de Dieu dans le monde. Ce postulat est évidemment problématique. Il pourra aisément donner prise à son inversion sceptique : les phénomènes caractéristiques de l'interaction entre l'âme et le corps, la perception sensible et le mouvement spontané, ne sont guère plus intelligibles que l'action par laquelle Dieu, présent au monde, perçoit et contrôle les objets qui s'y trouvent. On reconnaît d'ailleurs l'écho inversé (peut-être intentionnel et ironique) de la comparaison newtonienne chez Hume, dans ce propos destiné à montrer combien le pouvoir de l'homme sur son propre corps lui est incompréhensible : "Si nous avions le pouvoir par un secret désir, d'écarter les montagnes ou de contrôler les planètes dans leur orbite, cette puissance étendue ne serait pas plus extraordinaire et ne dépasserait pas davantage notre compréhension ${ }^{4}$."

Ces difficultés peuvent conduire à accueillir avec une certaine méfiance la métaphore. Certains commentateurs s'attachant à décrire les conceptions newtoniennes de l'espace y invitent d'ailleurs assez directement, estimant qu'on peut, et même qu'on doit, se dispenser des considérations métaphysiques pour comprendre ces conceptions ${ }^{5}$.

3. «Tout ce qu'on dit de Dieu est pris de quelque comparaison avec les choses humaines ; mais ces comparaisons, quoiqu'elles soient très imparfaites, en donnent cependant quelque faible idée " (I. Newton, Principes Mathématiques de la Philosophie Naturelle, trad. Marquise du Châtelet, Paris, 1759, Livre III, Scholie Général).

4. D. Hume, An Enquiry concerning Human Understanding, éd. P. H. Nidditch, Oxford, Clarendon Press, 1996, section 7, p. 65: "Is there any principle in all nature more mysterious than the union of soul with body ; by which a supposed spiritual substance acquires such an influence over a material one, that the most refined thought is able to actuate the grossest matter? Were we empowered, by a secret wish, to remove mountains, or control the planets in their orbit, this extensive authority would not be more extraordinary, nor more beyond our comprehension. »

5. Voir en particulier S. Toulmin, "Criticism in the History of Science: Newton on Absolute Space, Time and Motion " ( I \& II), Philosophical Review, 1959, 68, pp. 1-29 et 203-227. Toulmin réagit à ce qui lui apparaît être, depuis les travaux de E. Burtt et A. Koyré, une focalisation excessive des interprètes sur les aspects métaphysiques de la pensée newtonienne. Il considère avec circonspection les propos newtoniens sur Dieu et l'espace, ou ceux apparentés de More et Raphson. Loin de constituer des fondations métaphysiques, ces " superstructures théologiques " ressemblent plutôt à des "excroissances de stuc » ajoutées à la décoration d'un palais baroque.

Revue philosophique, $\mathrm{n}^{\circ} 1 / 2014$, p. 47 à p. 72 
Il faut remarquer également que le sensorium dei fut très inégalement apprécié par les disciples newtoniens du XvIII siècle. Tous ne partagent pas l'enthousiasme de Joseph Addison qui y voyait « la plus sublime manière de parler de l'espace ${ }^{6} »$. Par exemple, l'influente exposition de Newton rédigée par Henry Pemberton n'en fait pas mention, et celle de Colin McLaurin ${ }^{7}$ ne l'évoque qu'avec circonspection. Il est aussi assez symptomatique que Jean-Paul Marat, le traducteur français de l'Optique de Newton, ait gommé systématiquement dans sa traduction tous les passages où le mot sensory apparaissait ${ }^{3}$.

Si l'expression est aujourd'hui reconnue comme un identifiant fort de la «métaphysique newtonienne », c'est certainement en raison de la place centrale qu'elle occupe dans la polémique entre Leibniz et Samuel Clarke, dans les années 1715-1716 . Leibniz, entre plusieurs énoncés newtoniens lui paraissant témoigner d'une régression dangereuse de la théologie naturelle en Angleterre, avait commencé par considérer le sensorium dei comme une sorte de lubie ou d'aberration à la fois sémantique et philosophique, avant de comprendre, aux réactions de Clarke, l'attachement de ses adversaires à cette notion. L'expression devint dès lors l'un des points nodaux de la polémique, sur lequel se cristallisèrent un ensemble de questions cruciales pour les deux auteurs. Quel genre d'éclairage la philosophie naturelle donne-t-elle sur la cause première ? Quel genre de métaphysique convient à la promotion d'une juste idée de Dieu ? Leibniz voit les Anglais dominés par le démon d'une philosophie barbare et idolâtre, qui imagine Dieu substantiellement présent au monde, immanent

6. « But the noblest and most exalted Way of considering this infinite Space is that of Sir Isaac Newton, who calls it the Sensorium of the Godhead. Brutes and Men have their Sensoriola, or little Sensoriums, by which they apprehend the Presence and perceive the Actions of a few Objects, that lie contiguous to them. Their Knowledge and Observation turns within a very narrow Circle. But as God Almighty cannot but perceive and know every Thing in which he resides, Infinite Space gives Room to Infinite Knowledge, and is, as it were, an Organ to Omniscience » (Spectator, 9 juillet 1714, 565, p. XXX).

7. H. Pemberton, A View of Sir Isaac Newton's Philosophy, Londres, 1728 ; Colin McLaurin, An Account of Sir Isaac Newton's Discoveries, Londres, 1748, Book II p. 383-6. En revanche George Cheyne reprend, sans très bien le comprendre, le thème du sensorium divinatis dans la partie more geometrico de ses Philosophical Principles of Religion Natural and Reveal'd, $4^{\text {th }}$ éd. Londres 1733-4, pp. 53-54 de la seconde partie "The Nature and Kinds of Infinites".

8. Voir I. Newton, Optique, Traduit de l'anglais par Jean-Paul Marat, Paris, 1787 (réédition M. Blay, Paris, Christian Bourgois, 1989).

9. Sur la controverse, voir E. Vailati, Leibniz and Clarke: A Study of their Correspondence, Oxford, Oxford University Press, 1997, et H. Bernstein, "Leibniz and the Sensorium Dei », Journal of the History of Philosophy, 1977, 15, 2, pp. 171-182.

Revue philosophique, $\mathrm{n}^{\circ} 1 / 2014$, p. 47 à p. 72 
à celui-ci, et intervenant continuellement pour reformer la cohérence des parties et maintenir les planètes sur leurs orbites. Sous couvert de défendre la théologie naturelle, cette compréhension de l'omniprésence divine en menace l'idée même : elle ferait de Dieu un être asservi au monde, doté « d'organes » corporels ou quasi corporels, ou bien encore une anima mundi - en somme elle nous ferait basculer du côté d'une forme de panthéisme, et, de là, à la représentation athée d'un univers matériel animé de l'intérieur, éternel et auto-suffisant. Clarke, en retour, adresse à Leibniz et à l'esprit d'un certain cartésianisme dont il est solidaire une critique exactement symétrique : à vouloir défendre l'idée d'un Dieu supra-mondain qui, bien loin d'habiter et de diriger par son action le monde créé, se contente de le soumettre aux lois de la causalité efficiente et à un déterminisme inexorable, le tenant de l'harmonie préétablie ferait le jeu des athées qui pourront trop aisément se dispenser de la seconde partie de l'histoire racontée par Leibniz, et concevoir tout ce qui advient dans le monde et l'action humaine comme le produit d'une nécessité aveugle. Dans les deux cas, un épouvantail est agité, une caricature de la doctrine adverse, mais qui en montre peut-être une pente possible. La polémique n'échappe donc sans doute pas au procès d'intention, mais il est clair qu'elle manifeste aussi un différend profond, qu'aucun effort de persuasion ne pouvait réellement résoudre.

Dans ce débat, Clarke - un philosophe et théologien reconnu, qui a lui-même écrit sur l'existence et les attributs de Dieu ${ }^{10}-$ n'intervient pas seulement pour son compte, car d'emblée c'est l'œuvre et les dits de Newton qu'il s'agit de défendre. Leibniz sait par la princesse Caroline que Newton lit et peut-être supervise les réponses de Clarke $^{11}$. Newton lui-même ne souhaite pas intervenir publiquement dans la controverse métaphysique, mais l'on peut mesurer l'importance qu'il lui accorde à l'examen de multiples brouillons d'une lettre à Conti (l'un des médiateurs de la correspondance) et de certaines corrections du texte des Queries de l'Opticks dans la seconde édition anglaise de 1717, manifestement dictés par la volonté de répondre aux critiques leibniziennes ${ }^{12}$.

10. Voir S. Clarke, A Demonstration of the Being and Attributes of God, Londres, 1705.

11. D'après une lettre de Caroline à Leibniz de janvier 1716. Voir A. Koyré \& I. B. Cohen « Newton and the Leibniz-Clarke Correspondence, with notes on Newton, Conti and Des Maizeaux ", Archives Internationales d'Histoire des Sciences, 1962 , 15, pp. 63-126.

12. Les brouillons de la lettre à Conti sont reproduits en appendice de A. Koyré \& I. B. Cohen, art. cit., supra n. 11. 
Tout ceci invite à s'interroger sur la place que le sensorium dei occupe dans l'économie de la pensée newtonienne. Quels furent précisément le contexte et les intentions qui présidèrent à son introduction ? L'image était-elle appelée à rendre compte de la nature de l'espace infini, celle de Dieu, ou de celle de sa présence aux choses ? L'énoncé relève-t-il de la théologie ou de la métaphysique ? A-t-il aussi des implications pour la physique ? Pourquoi enfin, en dépit du caractère notoirement incompréhensible de l'interaction physique à l'œuvre dans la perception humaine, Newton en est-il venu à penser que la perception humaine des corps était un modèle instructif pour aborder la question métaphysique de l'omniprésence divine ? Cette dernière question nous conduira à examiner les considérations newtoniennes sur la perception visuelle consignées dans les carnets de notes de Cambridge des années 1660, dans le contexte des toutes premières recherches optiques de Newton. Ces textes méconnus permettent de jeter un jour neuf sur la source originelle de la métaphore, et ils témoignent de l'unité philosophique profonde qui chez Newton vient connecter ces champs d'investigation physique presque entièrement distincts que sont l'optique et la mécanique rationnelle.

\section{Le contexte métaphysique et religieux}

Si les occurrences textuelles explicites du sensorium dei sont rares dans le corpus newtonien et interviennent assez tardivement, l'énoncé se rattache clairement à une réflexion métaphysique sur les liens entre Dieu et l'espace entreprise anciennement (dès l'époque du $D e$ gravitatione $^{13}$ ), et qui se poursuit jusqu'aux Queries de l'Optique et aux différentes versions du Scholie Général des Principia. Cette réflexion pose de multiples problèmes. Son lien avec la notion d'espace absolu, telle que Newton l'expose dans le livre I des Principia, est difficile à établir. Il est douteux que les thèses ontologiques de Newton sur l'omniprésence divine soient directement à l'origine du concept physique d'espace absolu, dont on peut penser qu'il s'impose en vertu de nécessités internes aux considérations physico-mathématiques de Newton ${ }^{14}$. La chronologie des textes interdit de penser qu'elles puissent

13. Voir I. Newton, « De Gravitatione et aequipondio fluidorum », in I. Newton, Unpublished Papers of Isaac Newton, traduit et édité par A. R. Hall et M. B. Hall, Cambridge, Cambridge University Press, 1962 ; et pour une traduction française, I. Newton, De la Gravitation, éd. F. De Gandt, Paris, Gallimard, 1995.

14. C'est là la thèse principale de l'important article de S. Toulmin (art. cit supra, note 5).

Revue philosophique, $\mathrm{n}^{\circ} 1 / 2014$, p. 47 à p. 72 
à l'inverse en être dérivées ${ }^{15}$. Se pose aussi le problème des sources de la position métaphysique de Newton : l'influence de Henry More, d'Isaac Barrow, de Pierre Gassendi (via ses disciples anglais comme Walter Charleton) mais aussi celle d'auteurs anciens (les atomistes, la Kabbale, le néo-platonisme, la scolastique) furent très discutées dans l'historiographie ${ }^{16}$. Il semble que ces influences, quand bien même seraient-elles établies avec certitude, n'ôtent rien à l'originalité de la position newtonienne.

Ce que l'on peut dire de certain, car cela se manifeste immédiatement à la lecture du De gravitatione, est l'importance de la confrontation critique avec Descartes et avec l'identification cartésienne du corps et de l'étendue. Contre Descartes et contre un pan important de la tradition scolastique, Newton refuse de faire de l'espace un attribut de la création divine, qu'il s'agisse d'en faire une pure relation entre les choses créées ou l'attribut essentiel des corps. L'espace n'est pas rapporté à la création mais directement à l'existence du Créateur, dont il est, écrit Newton, "l'affection » ou « l'effet émanant ". Leibniz dans sa correspondance avec Clarke insiste sur le danger que fait courir à la religion naturelle une position qui apparemment consiste soit à faire de Dieu un être étendu, c'est-à-dire divisible, quantifiable et passif, soit à supposer qu'un être incréé, éternel et infini existe à côté de Dieu et indépendamment de lui. L'ontologie newtonienne du De gravitatione et d'un manuscrit plus tardif intitulé Tempus et Locus $^{17}$ permet cependant, jusqu'à un certain point, d'échapper à cette

15. La datation du De Gravitatione est ici un élément important du débat. Les premiers éditeurs anglo-saxons datent le manuscrit de la fin des années 1660. L'ouvrage de J. T. Dobbs, The Janus face of a Genius (1991) a pour un temps contribué à accréditer l'idée que le texte aurait été composé à une époque beaucoup plus proche de la rédaction des Principia, vers 1684 ; de nouveaux arguments avancés par J.-A. Ruffner (« Newton's De gravitatione: a review and reassessment », Archive for History of Exact Sciences, 2012, 66, 3, pp. 241-264). semblent pourtant confirmer la datation originelle des Hall.

16. A. Koyré (From the Closed World to the Infinite Universe, New York, Harper Torchbook, 1958) affirme l'étroite connexion entre Newton, More et Raphson. J. E. Mc Guire (« Existence, Actuality, and Necessity: Newton on Space and Time », Annals of Science, 1978, 35, 5, pp. 463-508) et B. Copenhaver (« Jewish Theologies of Space in the Scientific Revolution: Henry More, Joseph Raphson, Isaac Newton and their Predecessors ", Annals of Science, 1980, 37, 5, pp. 489-548), offrent différents arguments qui manifestent au contraire la distance entre ces auteurs. Voir aussi J. Carriero, "Newton on Space and Time: Comments on J-E. Mc Guire », in Ph. Bricker \& R. I. G. Hugues (eds.), Philosophical Perspectives on Newtonian Science, 1990, Cambridge, MIT Press, pp. 109-133 ; et pour une synthèse du débat, A. Janiack, Newton as Philosopher, Cambridge, Cambridge University Press, 2008, chap. V.

17. Présenté et traduit dans J.-E. Mc Guire, "Newton on Place, Time, and God: An Unpublished Source ", British Journal for the History of Science, 1978, 11, 2, pp. 114-129.

Revue philosophique, $\mathrm{n}^{\circ} 1 / 2014$, p. 47 à p. 72 
alternative. D'une part, l'espace, bien qu'incréé (et donc causalement indépendant de Dieu), ne pourrait exister sans Dieu, car il n'est rien d'autre que " l'amplitude » de sa présence - on ne peut donc aucunement penser qu'il le précède ou le concurrence. Comme le dit Newton, l'espace n'est posé que parce qu'un être est posé. Dès lors qu'un être existe, existe l'espace qui le sous-tend ou le contient ; et, partant, l'espace infini est la suite nécessaire de l'être infini qui est premier à exister. Paradoxalement, cette doctrine témoigne que l'espace, métaphysiquement considéré, n'est pas absolu. Il est bien relatif à l'existence d'un être ${ }^{18}$. En second lieu, contrairement aux thèses explicites de Henry More, Newton est attentif dans ses expressions les plus soignées à ne pas traiter l'espace comme une perfection, ou un attribut de Dieu, qui entrerait dans la définition de son essence. La spatialité de manière générale se définit comme une quantité d'existence, ce n'est pas un trait d'essence. C'est, écrit Newton, « l'affection commune de tout ce qui existe». En d'autres termes, dire de Dieu qu'il est omniprésent, qu'il est présent en substance dans chacune des parties de l'espace, ce n'est encore rien dire de sa nature essentielle. On ne peut certainement pas en tirer la conséquence que l'extension est un attribut de la substance divine, comme elle est l'attribut de la substance corporelle. À propos de l'usage parfois un peu relâché du terme « attribut » qu'on trouve chez Clarke, Newton interviendra auprès de Des Maizeaux, l'éditeur de la correspondance pour qu'il insère dans sa préface la précision suivante :

...lorsqu'il parle de l'Espace infini ou de l'Immensité et de la Durée infinie ou de l'Éternité, et qu'il leur donne, par une imperfection inévitable de langage, le nom de Qualités ou de Propriétés de la Substance qui est immense ou éternelle, il ne prétend pas prendre le terme de qualité ou de propriété dans le même sens que le prennent ordinairement ceux qui traitent de la Logique et de la Métaphysique lorsqu'ils les appliquent à la Matière, mais [...] par là, il veut seulement dire que l'Espace et la Durée sont des Modes d'existence dans tous les êtres; et des Modes infinis et des Conséquences de l'existence de la Substance qui est réellement, nécessairement et substantiellement toute-présente et éternelle ${ }^{19}$.

Ainsi, lorsque Newton traite des liens entre Dieu et l'espace, son ambition est d'offrir une conception intelligible de l'existence, plutôt que de la nature essentielle de Dieu. Il s'agit d'être dans le discours philosophique aussi proche que possible au sentiment religieux

18. Voir A. Janiak (op. cit. supra note 16), pp. 150 sqq. (« Newton’s Absolutism Revisited »), qui argumente ce point de façon persuasive.

19. Voir P. Des Maizeaux, Recueil de diverses pièces inédites sur la philosophie, la religion naturelle, l'histoire, les mathématiques, etc, par Mrs. Leibniz, Newton, Clarke et autres auteurs célèbres, Amsterdam, 1720, t. I, p. iv. 
naturel et commun, lequel ne peut satisfaire des manières de parler inintelligibles de certains scolastiques, reprises par de nombreux modernes, affirmant que Dieu est éternel mais qu'il n'est pas dans le temps, qu'il se tient dans un présent éternel (totum simul, nunc stans) ou qu'il est présent au monde virtuellement mais qu'il n'est pas proprement ou substantiellement dans le monde, qu'il est, comme l'affirmera Leibniz, supramundanus. Newton refuse de se payer de mots et d'accorder à Dieu un mode d'existence dont on n'aurait absolument aucune idée. Dire de Dieu qu'il est omniprésent, c'est dire qu'il est substantiellement présent au monde, bel et bien là, présent en chaque instant (sempiternel) et en chaque lieu. Il ne faut pas chercher à penser les choses autrement, sans quoi l'on en viendrait à exclure Dieu du rerum natura et à le penser comme un seigneur absent, ou un seigneur sans royaume.

\section{Occurrences et usages de la métaphore du sensorium}

Il fallait rappeler ce contexte spécifique du discours newtonien sur l'omniprésence divine pour mieux mettre en évidence les raisons qui conduisirent Newton à forger la métaphore du sensorium dei. La sensibilité « religieuse » de Newton l'incite à défendre l'idée d'une présence littérale de Dieu au monde, mais sa sensibilité «métaphysique » lui interdit de concevoir cette co-présence comme devant impliquer une quelconque forme d'unité substantielle (ou hylémorphique) de Dieu et du monde. Dieu n'est pas identique à l'espace qu'il occupe. Il ne l'est ni à la manière d'un corps cartésien, substantiellement étendu, diffusé, partes extra partes, ni à la manière d'une âme aristotélicienne, incarné dans l'existence spatiale des corps : il n'est pas l'âme du monde, et le monde n'est pas le corps de Dieu. La comparaison de l'espace avec le sensorium humain permettra de tenir précisément cette voie médiane qui affirme à la fois la présence substantielle de Dieu au monde et son entière indépendance à l'égard des corps qu'il traverse de sa présence.

Remarquons tout d'abord que c'est dans le même manuscrit Tempus et Locus, qui affirme le plus clairement l'idée selon laquelle l'espace est une affection de l'existence divine, qu'on trouve ce qui semble avoir été la toute première occurrence du sensorium dei.

Lidée de Dieu la plus parfaite est celle qui nous le représente comme une substance une, simple, indivisible, vivante et donnant la vie, existant nécessairement partout et toujours [...] ; une substance qui par sa propre présence discerne et gouverne toute chose, de même que la partie pensante de 
l'homme sent les espèces des choses déférées dans le cerveau et de là gouverne son propre corps ; [...] C'est là l'idée de l'être le plus parfait et une manière de le concevoir plus ardue ne fera nullement de Dieu un être plus parfait mais aura plutôt pour effet de le rendre suspect et de l'exclure du rerum natura ${ }^{20}$.

On reconnaît dans ce texte plusieurs éléments déjà soulignés au sujet de la théologie naturelle newtonienne : le refus des conceptions trop ésotériques de l'existence divine, l'affirmation de la présence effective de Dieu dans le monde, condition de sa souveraineté. Il est clair que la comparaison avec la perception humaine est sollicitée aussi dans ce contexte, pour souligner le rapport immédiat que Dieu entretient aux choses qu'il a sous sa vue et sous son pouvoir. Tout comme l'esprit percevant est immédiatement présent aux images qui sont véhiculées dans son cerveau, Dieu est immédiatement présent aux corps (et aux esprits) qui occupent l'espace. Nous reviendrons plus loin sur le sens de la doctrine de la perception humaine qui sert ici de modèle - retenons pour l'heure l'intention générale qui s'exprime ici : la métaphore du sensorium contribue à affirmer la présence littérale, et partant l'absolue souveraineté de Dieu sur le monde. Dieu est pensé comme Seigneur - pantokrator selon le mot du Scholium Generale. Il exerce son pouvoir de manière directe, sans délégation, sans médiateurs ou ministres, comme le fait l'âme en son sensorium, c'est-à-dire en son siège, ou comme le ferait un roi lorsque ses sujets sont directement en sa présence - autrement dit là où il siège, dans la salle du trône $e^{21}$. Le monde est le trône de Dieu : c'est-là le fond de la conviction religieuse de Newton. Il ne suffit pas de donner à Dieu ses attributs essentiels (l'unité, l'éternité, l'infinité, la perfection) pour se représenter correctement sa domination sur le monde. Pour entendre vraiment ce que signifie le mot Dieu, un terme qui exprime

20. Isaac Newton, Tempus et Locus, op. cit., supra note 17, notre traduction (nous soulignons).

21. On notera que la métaphore politique du trône, de la cour ou du palais pour caractériser le siège de l'âme est courante chez les auteurs que lit Newton. On la trouve par exemple dans ce passage très symptomatique du Natural History of Passions de Walter Charleton (Londres, 1674, sect. III pp. 61-62) : «I am very apt to judge, the imagination to be the Escurial, or Imperial palace of the Rational Soul, where she may most conveniently both receive all intelligences, from her Emissaries the senses, and give forth orders for government of the whole state of Man. That the whole corporeal soul should be possessed by the Rational, seems neither competent to her Spiritual nature, which is above Extensibility, nor necessary to her Empire over all: no more than it is necessary for a king to be present in all parts of his dominions at the same time. [...] This Queen of the Isle of Man hath her Court, and tribunal in the noblest part of the sensitive soul, the imagination. Made up of the select assembly of the most subtil spirits animal, and placed in the middle of the brain. »

Revue philosophique, $\mathrm{n}^{\circ} 1 / 2014$, p. 47 à p. 72 
seulement une relation de maître à sujet, il faut appréhender, plutôt qu'une essence, une manière d'exister, un mode de présence $\mathrm{e}^{22}$.

La dimension « religieuse »de l'intention newtonienne se double d'une dimension physico-théologique. Ici, il faut se tourner vers les deux textes importants des Questions de l'Optique où la métaphore apparaît de nouveau.

La query 28 (20 dans l'édition latine de 1706) examine l'hypothèse du fluide dense, ou matière subtile, supposé par certains (tels Descartes ou Huygens) pour expliquer les phénomènes physiques comme la transmission des mouvements de la lumière, ou la gravité. Newton donne plusieurs arguments pour le rejeter - et il finit par évoquer de manière un peu cryptique les tenants antiques (Grecs et Phéniciens) du vide et des atomes qui devaient donc attribuer « tacitement » la gravité à une autre cause que la matière dense. Cette affirmation est suivie de considérations selon lesquelles, la tâche de la physique étant de déduire les causes des effets manifestes, sans faire d'hypothèses, elle est potentiellement en mesure de nous faire remonter jusqu'à la cause première, en particulier lorsqu'il s'agit de donner l'explication de phénomènes qui révèlent directement l'influence d'un dessein intelligent, mais aussi lorsqu'il s'agit de répondre à la question de la cause de la pesanteur. Newton en vient alors à demander

Ne paraît-il pas, par les phénomènes, qu'il y a un être incorporel, vivant, intelligent, tout présent? Qui dans l'espace infini comme si c'était dans son sensorium, voit intimement les choses en elles-mêmes, les aperçoit, les comprend entièrement et à fond, parce qu'elles lui sont immédiatement présentes : desquelles choses il n'y a que les images seules qui étant portées par les organes des sens dans le lieu étroit de nos sensations puissent être aperçues par ce qui sent et pense en nous ${ }^{23}$.

22. Le Scholie Général des Principia défend précisément cette thèse, mais en suscitant d'autres ressources rhétoriques et conceptuelles, sans recourir au modèle du sensorium humain. Newton s'appuie sur la sémantique du mot Dieu, un terme par lequel nous marquons notre relation à un souverain ; voir Isaac Newton, Principes mathématiques, op. cit., Scholie Général, vol. II, pp. 175-176 : «Cet Être infini gouverne tout, non comme l'âme du monde, mais comme le Seigneur de toutes choses. Et à cause de cet empire, le Seigneur-Dieu s'appelle Pantokrator, c'est-à-dire, le Seigneur universel. Car Dieu est un mot relatif et qui se rapporte à des serviteurs : et l'on doit entendre par divinité la puissance suprême non pas seulement sur des êtres matériels, comme le pensent ceux qui font Dieu uniquement l'âme du monde, mais sur des êtres pensants qui lui sont fournis. Le Très-haut est un Être infini, éternel, entièrement parfait : mais un Être, quelque parfait qu'il soit, s'il n'avait pas de domination, ne serait pas Dieu. »

23. Newton, Optique, trad. P. Coste, Paris 1720, p. 445. L'histoire complexe de ce texte est rapportée dans A. Koyré \& I. B. Cohen : "The case of the Missing tanquam. Newton, Leibniz \& Clarke », Isis, 1961, 52, 4, pp. 555-566. Le texte latin 
Dans la Question 31 on trouve une seconde occurrence du sensorium, plus marquée encore par la perspecive physico-théologique. Après avoir évoqué l'uniformité et le dessein qui paraissent dans le corps et les organes des animaux, Newton conclut :

vous conviendrez que tout cet artifice ne peut être que l'effet de la sagesse et de l'intelligence d'un Agent puissant, et toujours vivant, qui, par cela qu'il est présent partout, est plus capable de mouvoir par sa volonté les corps dans son sensorium uniforme et infini, et par ce moyen de former et réformer les parties de l'univers que nous ne le sommes par notre volonté, de mettre en mouvement les parties de notre propre corps ${ }^{24}$.

On retrouve bien dans ces deux Questions le thème religieux d'un Dieu pleinement actif et souverain, mais l'on voit aussi plus clairement comment ce thème est potentiellement mobilisable dans le contexte de la physique newtonienne, au sein d'un discours causal qui prendrait en charge d'une part une explication directe de l'attraction, et d'autre part une justification de la stabilité de l'univers, pour laquelle il faut supposer non seulement une sage disposition des choses au moment de la Création, mais aussi des actes périodiques de « réforme " capables de compenser les effets entropiques de la dissipation des mouvements dans l'univers infini ${ }^{25}$. Solliciter la toute puissance de Dieu directement pour répondre à des questions ouvertes de physique constitue évidemment une démarche qui peut apparaître contraire à l'ethos du philosophe naturel. Newton ne fait d'ailleurs que suggérer cette option, sans jamais renoncer - en particulier pour l'explication de l'attraction - à la recherche de possibles causes physiques, tels qu'un éther très ténu et très élastique, un fluide électrique, etc. ${ }^{26} \mathrm{Il}$ convient toutefois de remarquer que le phénomène

initialement transmis à l'éditeur et qui, semble-t-il, figure encore dans l'exemplaire que Leibniz possédait ne comportait pas la clause atténuante du tanquam. Newton l'avait modifié dès 1706, par la substitution du feuillet concerné, mais le feuillet fut communiqué à l'éditeur tardivement, alors que plusieurs exemplaires avaient déjà été imprimés. Le texte des Questions fut de nouveau modifié et augmenté dans la seconde édition anglaise de 1717, où la question 20 est devenue 28 .

24. Optique, ibid. question 31, p. 491

25. Voir les quatre lettres de Newton à Richard Bentley de 1692-3, dans lesquelles Newton, sollicité par le philologue qui prépare pour ses Boyle Lectures une Confutation of Atheism, précise les enjeux physico-théologiques de ses découvertes, notamment s'agissant des conditions initiales et des actions de réforme périodiques qui président à l'ordonnancement du monde. Cf. Newton's Papers and Letters on Natural Philosophy and related Documents ed. I. B. Cohen, Cambridge, Harvard University Press, 1978, pp. 269 sqq.

26. Sur cette quête des causes physiques de l'attraction, voir en particulier l'exposé très clair de E. McMullin, Newton on Matter and Activity, Notre Dame, University of Notre Dame Press, 1978, chap. IV.

Revue philosophique, $\mathrm{n}^{\circ} 1 / 2014$, p. 47 à p. 72 
même de l'attraction impose une condition extrêmement stricte à la recherche causale, car il s'agit d'expliquer une force qui opère sur les corps non pas en proportion de leur surface - comme le font généralement toutes les causes mécaniques - mais en proportion de leur masse solide ${ }^{27}$. En somme la cause de l'attraction, quelle qu'elle soit, devra être en mesure de pénétrer les corps intimement, dans toute leur substance. Le texte des Queries et la métaphore du sensorium dei servent donc une fin physico-théologique bien précise lorsqu'ils affirment que Dieu lui-même est une telle cause pénétrante, présente en tout lieu, y compris dans ceux que les corps occupent, qu'il a et conserve quoi qu'il arrive le pouvoir de mouvoir les corps à sa guise, qu'il n'est pas soumis à la nécessité aveugle du mécanisme, et peut par fiat changer le cours des choses naturelles. Dieu pourrait se dispenser de tout intermédiaire physique et maintenir par sa seule volonté les planètes sur leurs orbites, en proportion exacte de leur masse et de leurs distances. Cette option métaphysique est ouverte. L'intervention active de Dieu dans la Création pourrait aussi être à la source du mouvement des comètes dont la masse et la trajectoire seraient dosées de telle sorte qu'elles rétabliraient à chacun de leur passage l'ordre du système par leurs effets gravitationnels.

Il convient de remarquer que cette insistance sur l'intervention active de Dieu requise par la théologie physique newtonienne conduit à transformer de manière assez sensible la métaphore du sensorium. Au sens strict le sensorium est le lieu de la présence sensible de l'esprit, l'espace de la perception. Mais dans les Questions, et particulièrement dans la Question 31, le sensorium est mentionné au sujet de l'action motrice volontaire : Dieu, nous dit Newton, est plus capable de mouvoir les corps dans son sensorium infini que nous ne sommes par notre volonté capables de mouvoir les parties de notre corps.

Ce propos marque un double déplacement dans l'usage de la métaphore. Déplacement, car l'espace universel ou le sensorium dei évoqué dans la Question 31 n'est plus mis en regard du seul sensorium humain mais du corps dans sa globalité. Déplacement ou

27. Voir I. Newton, Principes Mathématiques, op. cit., Scholie Général, p. 179 : "J'ai expliqué jusqu'ici les phénomènes célestes et ceux de la mer par la force de la gravitation, mais je n'ai assigné nulle part la cause de cette gravitation. Cette force vient de quelque cause qui pénètre jusqu'au centre du Soleil et des planètes, sans rien perdre de son activité ; elle n'agit point selon la grandeur des superficies (comme les causes mécaniques), mais selon la quantité de la matière ; et son action s'étend de toutes parts à des distances immenses, en décroissant toujours dans la raison doublée des distances. » 
écart, parce que la comparaison manifeste aussi une différence de degré : Dieu est plus capable d'agir sur les corps dans son sensorium infini, que nous ne sommes nous-mêmes en mesure de contrôler notre corps. La maîtrise volontaire que nous avons de nos actions corporelles, via les muscles, les nerfs et les esprits animaux, est loin en effet d'être aussi complète et parfaite que celle que le Dieu, créateur de toute chose et intimement présent à toute chose, possède sur le monde.

Il semble que l'on ait atteint ici les limites de la métaphore. En faisant dériver l'analogie perceptive du côté du corps et de son animation volontaire, Newton paraît s'approcher dangereusement de l'écueil du panthéisme. Le corps que nous mouvons est beaucoup plus étroitement nôtre que les images perceptives dépeintes dans le sensorium. Doit-on dire que l'univers est le corps de Dieu ? C'est très certainement pour se prémunir contre cette interprétation scabreuse, à laquelle Leibniz n'avait pas hésité à donner voix, que Newton ajoute dans la seconde édition anglaise de 1717 de la Question 31 d'importantes précisions à la suite du texte précédent :

Nous ne devons pourtant pas considérer le Monde comme le Corps de Dieu, ni les différentes parties du monde comme autant de parties de Dieu. Dieu est un être uniforme, sans organes, sans membres ou parties ; et toutes les différentes parties du monde étant ses créatures, lui sont subordonnées, et dépendent entièrement de sa volonté. Il n'est non plus leur âme que l'âme de l'homme est l'âme des images, qui par les organes des sens sont portées dans le lieu de ses sensations, où elle les aperçoit par sa présence immédiate sans l'intervention d'aucune troisième chose. Les organes des sens n'ont pas été formés pour mettre l'âme en état d'apercevoir les espèces ou images des choses dans son sensorium, mais seulement pour les conduire en cet endroit-là ; et Dieu n'a pas besoin de pareils Organes, parce qu'il est présent partout aux Choses mêmes ${ }^{28}$.

Dieu est intimement présent au monde, il le perçoit et l'anime, mais il n'en est pas l'âme, pas plus que l'âme humaine n'est l'âme des images véhiculées dans son sensorium. C'est là le dernier mot de Newton sur la manière de comprendre cette métaphore énigmatique. Clarke, qui avait apporté des précisions analogues à Leibniz, s'était vu opposé une fin de non recevoir et une perplexité non feinte sur l'étrange langage utilisé : que veut-on dire lorsqu'on dit que l'esprit est présent aux images mais qu'il n'en est point l'âme ? Quelle théorie de la perception humaine et quelle théorie de l'action motrice volontaire étaient susceptibles de nourrir une telle conception ? C'est la question à laquelle nous nous proposons de répondre à présent.

28. Optique, question 31, p. 491.

Revue philosophique, $\mathrm{n}^{\circ} 1 / 2014$, p. 47 à p. 72 


\section{Le sensorium humain et la théorie newtonienne de la perception}

Il est à peu près certain que Newton a découvert le terme technique de sensorium chez Henry More. Dans son Notebook d'étudiant à Cambridge, dans lequel il consigne ses toutes premières recherches philosophiques (entre 1661 et 1665) il transcrit un passage de l'Immortality of the Soul (1659) dans lequel More évoquait la question de la localisation physique du sens commun, ce common sensorium, qu'il définissait comme l'organe interne par lequel l'âme " non seulement appréhende les objets des sens externes mais aussi imagine, raisonne et librement commande et détermine les esprits à aller dans quelque partie du corps qu'elle veut ${ }^{29}$ ". Sous l'entrée de son Notebook «Of sensation "Newton recopie soigneusement les différentes têtes de chapitres qui, dans l'ouvrage de More, traitent de ce siège du sens commun : «Le sensorium commun est ou bien 1) Le corps tout entier 2) l'orifice de l'estomac 3) le cour 4) le cerveau 5) les membranes 6) le septum lucidum 7) une partie du corps très petite et parfaitement solide 8) le conarion 9) le concours des nerfs aux alentours du $4^{\mathrm{e}}$ ventricule du cerveau 10) Les esprits animaux de ce quatrième ventricule ${ }^{30}$. »

Ce passage appelle quelques remarques. Il permet d'abord de comprendre le contexte originel qui fixe pour Newton le sens du terme sensorium. Dans la suite de son œuvre, Newton s'en servira toujours comme d'un terme abréviatif, qui renvoie bien au common sensorium décrit par More. Pour des raisons qui apparaîtront plus clairement bientôt, cet organe du sens commun est présenté, aussi bien par More que par Newton, comme le lieu privilégié des deux sortes d'interactions physiques entre l'âme et le corps : la perception et le mouvement volontaire.

L'idée selon laquelle l'âme siège non dans le corps tout entier, mais plus particulièrement là où s'exerce le sens commun doit être

29. H. More, The Immortality of the Soul, Londres, 1659, II, chap. VII, pp. 124-125.

30. Voir J. E. McGuire \& M. Tamny, Certain Philosophical Questions. Newton's Trinity Notebook, Cambridge, Cambridge University Press, 1999, [ci-après CPQ], fol. 33104 r, p. 382 ; H. More, Immortality of the Soul, op. cit., II, chap. IV, p. 103. On reconnaît dans les solutions proposées quelques unes des doctrines anciennes et modernes : le common sensorium est encore le coeur chez Hobbes comme il l'était chez Aristote ; c'est la glande pinéale ou conarion chez Descartes, le septum lucidum chez Digby. More lui-même adopte l'opinion que le siège du sensorium est le lieu de jonction des nerfs et de la moelle épinière, espace qu'il assigne au quatrième ventricule du cerveau, mais dont il se refuse à faire le siège exclusif de l'âme, remarquant qu'elle a le pouvoir de s'étendre dans le corps, et notamment, pour la vision, jusqu'à la rétine, le seul endroit où les images des corps se peignent avec leur couleurs.

Revue philosophique, $\mathrm{n}^{\circ} 1 / 2014$, p. 47 à p. 72 
replacée à l'époque moderne dans le contexte d'un intérêt renouvelé pour l'anatomie du cerveau et la physiologie nerveuse. L'idée d'une centralisation cérébrale des fonctions de perception et de contrôle moteur semble de mieux en mieux fondée. L'observation des amputés qui sentent de la douleur dans le membre qu'ils n'ont plus, et de ceux dont les nerfs ont été ligaturés et dont les organes, intacts, cessent d'être sensibles ou fonctionnels, confirme l'importance du système nerveux central pour la sensibilité et la motricité. L'anatomie met en évidence la convergence des nerfs dans le cerveau. Tout laisse donc supposer que le cerveau, ou une certaine région de celui-ci, joue un rôle important pour l'activité traditionnellement assignée au sens commun, la fusion des informations sensorielles en provenance des différents sens, ou en provenance des différents organes du même sens. Dans ce mouvement d'idées, la physiologie cartésienne joue certainement un rôle d'impulsion. La centralisation des fonctions sensorielles et motrices (mouvement volontaire et involontaire) à l'interface de la glande pinéale est clairement affirmée dans le Traité de l'Homme, puis dans les Passions de l'âme. Le détail de l'explication, et particulièrement le rôle assigné à cette glande, se voit en revanche sérieusement critiqué, par exemple dans les travaux de Willis ou de Stenon.

Newton lui-même prend une option déterminée sur ces questions à l'occasion de ses propres recherches psycho-physiologiques sur la vision binoculaire. Dans le Notebook de Cambridge et dans un manuscrit contemporain intitulé Of Colours, l'anatomie du système oculaire et les expériences de vision contrainte lui paraissent attester l'existence d'une image cérébrale formée à la seconde jonction des deux nerfs optiques. Newton suit la structure générale (sinon le détail) de l'analyse " cérébraliste » cartésienne : les deux yeux participent ensemble à la vision et contribuent à former une image unique dans le cerveau. La vision ne s'effectue pas sur la rétine comme le croyait encore More, et elle n'est pas fondamentalement monoculaire comme l'avaient cru Gassendi et Charleton. Le cerveau a donc une fonction déterminée au regard de l'activité de synthèse des images en provenance des deux yeux. Cette synthèse s'accomplit comme effet d'une superposition physique des images, elle-même résultant d'une fusion des véhicules nerveux.

Un ensemble de questions soulevées par Newton au sujet de la perception visuelle permet de se faire une idée du statut perceptuel de cette image cérébrale :

Questionl : Pourquoi les objets n'apparaissent-ils pas inversés ? Réponse : l'esprit ou l'âme ne peut juger que l'image dans le cerveau est inversée, à moins de percevoir aussi les choses extérieures auxquelles elle pourrait 
comparer leur image. 2. Pourquoi les objets apparaissent-ils à l'extérieur du corps ? Réponse : parce que dans l'image dessinée dans le cerveau par la vue, l'image du corps est placée au milieu des images des autres choses et elle est mue à notre commande de telle sorte qu'elle se rapproche ou s'éloigne de ces images etc. 3. Mais pourquoi ces objets ne sont-ils pas jugés être dans le cerveau ? Réponse : parce que l'image du cerveau n'y est pas dépeinte, et parce que le cerveau n'est pas perçu par l'âme, n'étant pas en mouvement : l'âme probablement ne perçoit les corps que par le moyen de leur mouvement. Mais si le cerveau était perçu avec les images en lui, nous penserions voir un corps comme le cerveau englobant et comprenant en lui nous-mêmes, les étoiles et tous les autres objets visibles ${ }^{31}$.

Les réponses apportées ici à quelques-unes des questions typiques soulevées par l'analyse moderne de la vision suggèrent une adhésion à une forme assez brute de ce que nous appellerions aujourd'hui le réalisme indirect, « représentationnel ${ }^{32}$ » : nous ne voyons pas directement le monde extérieur, comme le suppose le réalisme de sens commun, nous n'avons accès à lui qu'au terme d'une inférence implicite qui s'appuie sur ce qui seul nous est donné directement : les images qui se forment dans notre cerveau, objets immédiats de la conscience perceptive. La réponse de Newton au fameux problème de "l'inversion " rétinienne ou cérébrale est symptomatique de cette position. Pour Newton, le problème de l'inversion n'est apparemment qu'un faux problème : nous ne pouvons pas comparer la situation de l'image cérébrale et celle des objets extérieurs pour affirmer l'inversion, car nous n'avons d'accès visuel qu'à la première, et c'est donc de manière intrinsèque, en comparant des éléments internes à cette image, et non pas de manière extrinsèque que peuvent se déterminer les directions de l'espace vécu. La réponse newtonienne à la seconde question complète cette analyse : nous ne saurions pas que les choses que nous voyons sont hors de nous si nous n'apprenions pas d'abord à reconnaître notre propre corps dans notre champ visuel et ainsi à juger que celui-ci se situe hors de celui-là. La troisième question et la réponse que Newton y apporte sont encore plus révélatrices de la position philosophique sous-jacente. Pour rendre compte du fait que nous ne percevons pas le cerveau sur le fond duquel les images cérébrales se découpent, Newton suppose que c'est par le mouvement qui les constitue que les images sont appréhendées consciemment. Ainsi l'image cérébrale n'est-elle pas identique en tout à la pensée qu'elle suscite. Ce qui est

31. CPQ, fol. $86130 v$, pp. 451-452 (« of the soul »).

32. Nous nous appuyons dans ce paragraphe sur des analyses déjà présentées dans notre article : P. Hamou "Quelle théorie de la vision pour l'optique newtonienne? ", Lumières, 2004, 4 (Regards sur l'Optique de Newton, 1704-2004), pp. 33-64.

Revue philosophique, $\mathrm{n}^{\circ} 1 / 2014$, p. 47 à p. 72 
mouvement en elle est assurément « traité » par l'esprit pour devenir lumière et couleur dans l'expérience mentale ${ }^{33}$. Il reste, et c'est le point fondamental ici, que c'est bien l'image dans le cerveau et non pas le monde extérieur qui fait l'objet de la conscience perceptive : cette image est bel et bien perçue là où elle est, dans l'espace du sensorium où nos sens, ou éventuellement notre imagination, la dépeignent. Ce qui l'atteste ici particulièrement est l'affirmation contrefactuelle selon laquelle le cerveau lui-même avec ses circonvolutions pourrait être vu comme une sorte de corps englobant les corps perçus, si son immobilité relative ne le rendait de facto transparent à la conscience.

Cette doctrine newtonienne de la perception est apparue passablement « naïve » à certains commentateurs ${ }^{34}$. Elle appartient sans doute au genre de doctrines qu'on pourrait juger vulnérables au «paradoxe de l'homoncule » que la philosophie contemporaine de l'esprit a maintes fois dénoncé : dans cette doctrine de la perception n'est-on pas conduit à supposer qu'au cœur de notre cerveau un petit homme ouvre les yeux et contemple ou inspecte une image ? Mais comment lui-même verrait-il sinon par une autre image ? De manière générale, comment échapper au sentiment de circularité lorsque pour expliquer la perception des corps extérieurs, on convoque la « perception » d'un autre objet physique, l'image cérébrale ? John Norris, un contemporain anglais de Newton et de Locke, introducteur du malebranchisme en Angleterre dans son Essai pour la théorie du monde intelligible, avait déjà formulé de telles critiques : selon lui, et selon l'enseignement authentique de Malebranche (et peut-être d'un Descartes bien compris), l'objet immédiat de la vision ne peut aucunement être une image, c'est un être intellectuel - « l'âme, écrit-il, ne contemple pas des images dans le cerveau ou sur la rétine comme si c'était des tableaux accrochés en quelque galerie fantastique ${ }^{35}$ ", elle n'a de rapport immédiat qu'aux idées.

La critique exprimée par Norris s'adresse en général aux « optical men », et ce terme est assez symptomatique parce qu'il révèle que l'opinion visée par le malebranchiste anglais, loin d'avoir été une simple idiosyncrasie newtonienne, était en fait assez largement répandue parmi les contemporains de Newton. En dépit de ses difficultés

33. Cette réduction des qualités secondes est l'un des mots d'ordre de la philosophie moderne auquel Newton ne déroge pas : il est difficile d'imaginer comment nous pourrions « voir » des couleurs dans l'espace opaque du cerveau.

34. Voir en particulier M. Tamny, « Newton, Creation, and Perception », Isis, 1979, 70, pp. 48-58.

35. J. Norris, An Essay towards the Theory of the Ideal or Intelligible World, Londres, 1701, vol. I, p. 207.

Revue philosophique, $\mathrm{n}^{\circ} 1 / 2014$, p. 47 à p. 72 
théoriques, un modèle " optique », ou picturaliste de la représentation (nous voyons le monde par la médiation de tableaux projetés dans notre tête) est largement diffusé et accepté en Angleterre, chez nombre d'auteurs représentatifs du climat d'opinion de cette époque. Nous pourrions le montrer à l'œuvre chez des auteurs comme Glanvill, Hooke, et peut-être Locke ${ }^{36}$. Et c'est précisément parce que ce modèle est bien reçu que Newton estime pouvoir s'appuyer sur son intelligence partagée pour faire entendre la métaphore du sensorium et de la présence sensible dans ses textes sur Dieu et l'espace.

Remarquons en second lieu qu'il ne faut peut-être pas céder trop vite à la suggestion selon laquelle ce modèle serait le fruit d'une philosophie naïve. En fait, s'il s'impose à la pensée anglaise du second $\mathrm{XVII}^{\mathrm{e}}$ siècle, c'est pour des raisons philosophiques assez profondes.

La première d'entre elle est la dépendance causale forte qu'on peut établir entre l'expérience perceptive et la présence factuelle des images cérébrales. Ici, ce sont les faits de la physiologie qui s'imposent à la théorie de la perception. Même si nous ne savons pas mieux ce que signifie percevoir lorsque nous appliquons le mot à une image dans notre tête que lorsque nous l'utilisons couramment pour parler de notre appréhension des choses au dehors, c'est pour ces auteurs un fait (prouvé par l'anatomie et l'expérimentation) que l'âme a besoin pour percevoir que des images (ou quelque chose comme des images) se soient formées dans l'œil, puis dans le cerveau. On pourrait de même évoquer des faits physiologiques analogues, qui permettent de dire que l'action motrice volontaire est causalement corrélée à des modifications ou des impulsions cérébrales capables de diriger le flux des esprits animaux vers les muscles et les membres ${ }^{37}$.

Cette dépendance est une vraie dépendance causale. Newton n'a aucune espèce de sympathie pour les explications occasionnalistes ou

36. Sur la prévalence de ce modèle en Angleterre, voir J. Yolton, Perceptual Acquaintance from Descartes to Reid, Minneapolis, University of Minesota Press, 1984. Yolton ne compte pas toutefois Locke au nombre des auteurs représentatifs de cette «perceptual opticks ».

37. Newton développe des considérations physiologiques approfondies sur le mouvement volontaire dans son Hypothesis explaining the properties of Light de 1675) (I. B. Cohen (ed.), Isaac Newton's Papers and Letters on Natural Philosophy and Related Documents, Cambridge, Harvard University Press, 1978, p. 184). Il y propose une manière de comprendre comment l'âme, sise dans le cerveau et à la jonction des nerfs, suscite le mouvement animal en envoyant dans les nerfs une substance susceptible de catalyser la mixtion entre un fluide élastique universel (l'éther) et les fluides et la substance des muscles. C'est ce mélange qui permet au muscle de se gonfler. Voir sur ce thème l'important article de R. Iliffe, "That puzzling problem'. Isaac Newton and the Political Physiology of the Self », Medical History, 1995, 39, pp. 433-458. 
« harmonique » de la perception ou de l'action motrice, qu'il juge des remèdes pires que le mal. Il ne met jamais en question le postulat de l'influence physique : dans la perception, il y a une causalité directe du corps sur l'esprit (et réciproquement une action de l'esprit sur le corps dans le mouvement volontaire). Cela est factuel et indiscutable, quand bien même cela serait-il incompréhensible ${ }^{38}$.

Par ailleurs, comme le montrent certaines expériences de fixation des objets lumineux, les images intérieures produites par les sens apparaissent douées d'un certain pouvoir de rémanence, de sorte que, conformément à un thème hobbesien, elles seraient susceptibles de persister à l'intérieur de nous, lors même que le monde extérieur serait annihilé. Comme l'écrit Hobbes, certains êtres naturels, ceux pour lesquels il y a de l'apparaître, ont cette propriété tout à fait singulière « d'avoir au-dedans d'eux le modèle de presque toute chose ${ }^{39}$ ". C'est là le signe d'une certaine indépendance causale, mais cette fois des images elles-mêmes au regard des corps extérieurs qui sont supposés leur avoir donné naissance. L'expérience du monde est pour ainsi dire préformée à l'intérieur de nous, dans la plasticité du sensorium. Certes les sensations sont produites par les choses extérieures, mais en principe rien n'interdit de penser qu'elles puissent naître à volets clos comme de purs produits de l'imagination. Il existe à cet égard une parenté fondamentale entre la sensation et l'imagination, et Newton, qui lit le De Corpore dans ses années de jeunesse, l'a toujours reconnue, mettant l'accent dans ses recherches sur la vision sur l'homogénéité et, partant, la possible confusion entre les fantasmes produits par la sensation et ceux produits par l'imagination ou la phancy ${ }^{40}$.

38. L'hostilité de Newton à l'harmonie préétablie et sa défense de l'influence physique sont clairement exprimées dans un des fragments manuscrits transcrits par A. Koyré \& I. B. Cohen, «Newton and the Leibniz-Clarke Correspondence, with notes on Newton, Conti and Des Maizeaux ", art, cit., supra n. 11 (Porstmouth Collection Cambridge University Library Add 3968, fol. 591). Newton y examine de manière critique les arguments leibniziens: "And whilst he applauds Eperimental [sic] Philosophy and exclaims against Miracles, he introduces an Hypothesis of Harmonia praestabilia which cannot be true without an incredible Miracle, and is contrary to the daily experience of all mankind. For all men find by experience that they can move their bodies by their will, and that they see heare and feel by means of bodies. "

39. Th. Hobbes, Elements of Philosophy. The First Section concerning Bodies, Londres, 1656, repr. in The English Works of Thomas Hobbes of Malmesbury, ed. by W. Molesworth, 11 vols., London, 1845, vol. I, chapitre XXV, art. 1, p. 389 : «Of all the phenomena or appearances which are near us, the most admirable is apparition itself, to phainestai, namely that some natural bodies have in themselves the pattern almost of all things, and other of none at all. »

40. Voir $C P Q$, pp. 387, 485-486. Voir aussi la lettre de Newton à Locke de 1691, Isaac Newton, Correspondence of Isaac Newton, op. cit, vol. III, letter 365, pp. 152-154.

Revue philosophique, $\mathrm{n}^{\circ} 1 / 2014$, p. 47 à p. 72 
Enfin, l'idée selon laquelle la perception se fait « dans notre tête ${ }^{41}$ » procède de l'usage plus ou moins tacite d'un principe « présentiste » ou « contiguïste » qui semble avoir été considéré par nombre d'auteurs comme inhérent à la logique de l'action causale. Ce principe, maintes et maintes fois formulé, depuis la première scolastique jusqu'à Hume, est énoncé ainsi par Samuel Clarke : "Une chose ne peut pas plus agir ou être agie là où elle n'est pas présente, qu'être là où elle n'est pas ${ }^{42}$. " La cause doit être au contact de son effet pour s'exercer. En d'autres termes : il n'y a pas d'action à distance, pas d'action sans co-présence substantielle, contiguïté ou compénétration de la cause et de l'effet. Le paradoxe veut que Newton, l'auteur qui passe pour avoir introduit la notion d'une force attractive s'exerçant « à distance », a pour sa part nié, et de la manière la plus véhémente $^{43}$, que les corps puissent avoir de manière inhérente le pouvoir

41. Cette croyance révèle, d'après Kant, un vice de subreption : « Encore bien que la plupart des hommes croient sentir la pensée dans la tête, c'est là toutefois un simple vice de subreption, qui consiste à prendre le jugement sur la cause de la sensation éprouvée en un certain endroit (du cerveau) pour la sensation de la cause en ce même endroit » (Lettre de Kant à Sommering, in E. Kant, Anthropologie, trad. Tissot, Paris, Ladrange, 1863, p. 443). Sur cette accusation, et sur l'héritage qu'elle révèle chez Kant d'une certaine métaphysique scolastique et cartésienne de la présence « virtuelle », voir l'article de Laurent Jaffro dans ce même numéro. On peut estimer que Newton, tout comme Locke (qui défend aussi une interprétation littérale de la présence spatiale de l'esprit) seraient restés imperméables à la critique. Voir J. Locke, An Essay concerning human understanding, 2.23, 21, éd. P. H. Nidditch, Oxford, 1975, p. 307 : «If it be said by any one that it cannot change place, because it hath none, for the spirits are not in loco, but ubi; I suppose that way of talking will not now be of much weight to many, in an age that is not much disposed to admire, or suffer themselves to be deceived by such unintelligible ways of speaking. "

42. "Nothing can anymore act or be acted upon, where it is not present than it can be where it is not ", Lettre du 16 janvier 1716 (seconde réponse de Clarke), in A. Robinet, (éd.) Correspondance Leibniz-Clarke présentée d'après les manuscrits originaux des Bibliothèques de Hanovre et de Londres, Paris, Puf, 1957, p. 48. Pour une présentation plus générale de ce principe voir T. Lennon, The Battle of the Gods and Giants. The Legacies of Descartes and Gassendi, 1655-1715, Princeton, Princeton University Press, 1993. Le principe présentiste (le refus de l'action à distance) est explicitement identifié par Dugald Stewart comme la source de la doctrine du sensorium dei, qu'il critique sur des bases analogues à celles de Kant (voir note précédente) dans ses Elements of Philosophy of the Human Mind, Londres 1792, pp. 82-83.

43. Voir la lettre de Newton à Richard Bentley du 25 février 1693. Nous donnons ici ce texte controversé dans la transcription normalisée du manuscrit original procurée par le site du Newton Project de l'Université du Sussex: http:// www.newtonproject.sussex.ac.uk/view/texts/normalized/THEM00258 (consulté le 15 octobre 2013) : "Tis unconceivable that inanimate brute matter should (without the mediation of something else which is not material) operate upon \& affect other matter without mutual contact; as it must if gravitation in the sense of Epicurus be essential \& inherent in it. And this is one reason why I desired you would not ascribe $\{$ innate $\}$ gravity to me. That gravity should be innate inherent \& essential $\}$

Revue philosophique, $n^{\circ} 1 / 2014$, p. 47 à p. 72 
d'agir les uns sur les autres à distance. Ce principe présentiste a une portée ontologique générale : il s'applique non seulement aux actions des corps entre eux, mais aussi aux actions des esprits les uns sur les autres, des esprits sur les corps et des corps sur les esprits. L'interdit qui pèse sur l'action à distance concerne donc aussi éminemment la perception, et particulièrement la perception visuelle qui bien qu'elle semble illusoirement nous faire voir les choses directement dans le monde et comme à distance, sans medium d'aucune sorte, ne nous fait en réalité percevoir que ce à quoi nous sommes intimement unis.

Sur l'ensemble de ces points une confrontation avec Descartes s'impose. En un certain sens Descartes peut être considéré comme la source théorique privilégiée des conceptions newtoniennes. La physiologie des sens présentée dans la Dioptrique ou le Traité de l'homme, défend de manière très persuasive l'idée que la perception requiert la formation d'images cérébrales dans un certain espace privilégié du cerveau identifié au "siège de l'imagination et du sens commun ${ }^{44}$ " - un espace qui se trouve être aussi le lieu à partir duquel s'effectuent les réactions sensori-motrices instinctives, et l'action motrice volontaire. Mais on sait aussi que Descartes a fortement combattu l'interprétation " naïve » selon laquelle, pour avoir un accès (indirect) au monde extérieur, il faudrait d'abord percevoir les images dans le cerveau, comme s'il s'agissait de petits tableaux ressemblants formés à l'intérieur de la tête. Dans la Dioptrique il indique que la dépendance de la perception à l'égard des images pourrait tout aussi bien être comprise sur la base d'un modèle sémiotique. L'image fonctionnerait comme un mot ou un signe d'institution arbitraire (quoique naturelle), capable d'exciter notre pensée à se représenter un objet sans entretenir aucune sorte de ressemblance avec celui-ci ${ }^{45}$. Descartes cepen-

to matter so that one body may act upon another at a distance through a vacuum without the mediation of any thing else by \& through which their action or force $\{$ may\} be conveyed from one to another is to me so great an absurdity that I believe no man who has in philosophical matters any competent faculty of thinking can ever fall into it. Gravity must be caused by an agent \{acting\}consta\{ntl\}y according to certain laws, but whether this agent be material or immaterial is a question I have left to the consideration of my readers. "

44. R. Descartes, Traité de l'homme, in R. Descartes, Euvres de Descartes, édités par C. Adam et P. Tannery (12 vol.), Paris, Vrin - CNRS, 1964-1976, vol. XI, p. 177 .

45. Voir R. Descartes, Dioptrique, in R. Descartes, Euvres de Descartes, op. cit., vol. VII, p. 112 : «Voyant que notre pensée peut facilement être excitée, par un tableau, à concevoir l'objet qui y est peint, il leur a semblé qu'elle pouvait l'être en même façon, à concevoir ceux qui touchent nos sens par quelque tableau qui s'en formasse en notre tête, au lieu que nous devons considérer qu'il y a plusieurs autres choses que des images, qui peuvent exciter notre pensée. »

Revue philosophique, $\mathrm{n}^{\circ} 1 / 2014$, p. 47 à p. 72 
dant n'opte pas de manière unilatérale pour ce modèle. Dans certains textes, il semble indiquer que l'image cérébrale fait bel et bien l'objet d'une inspection mentale, en particulier pour tout ce qui concerne sa grandeur, sa figure ou sa situation ${ }^{46}$. Il indique seulement que, tout comme pour les tableaux de perspective, les images dépeintes sur la glande pinnéale représentent moins par leur ressemblance que par l'existence d'un écart réglé dans la relation expressive qu'elles instaurent avec leur modèle.

Newton, il me semble, reconnaîtrait ces points tout aussi bien, et jusque-là il serait cartésien. L'écart avec la Dioptrique cartésienne se creuse en revanche lorsqu'on considère les conditions physiques de production de l'image cérébrale. Chez Descartes, l'image n'est pas coupée des objets qui lui ont donné naissance. Elle en reste à tout moment physiquement solidaire, via la matière du second élément qui, comme le bâton de l'aveugle, communique la pression lumineuse jusqu'à la rétine, et via les nerfs jusqu'aux surfaces internes du cerveau. Dans ce processus de transmission, l'image rétinienne ou l'image cérébrale ne sont donc que des moments, abstraits du processus causal pour les besoins de son analyse, mais non réellement détachés de lui. Si, contrairement aux apparences, il n'y a pas réellement d'action à distance dans la vision, c'est donc parce que voir c'est entrer littéralement en contact avec le monde visible. La cause prochaine de l'impression visuelle n'est pas dans la tête mais bien sur les corps vus, et dans les mouvements par lesquels ils viennent pour ainsi dire directement toucher nos facultés sensibles. Newton en revanche poursuit ses recherches sur la vision et la lumière dans une ambiance atomiste. À ses yeux, il est hautement vraisemblable que la matière n'occupe que de toutes petites portions d'un espace qui, pour le reste, est vide de tout corps. Le processus causal à la source de l'image cérébrale n'est donc pas supporté par l'existence continue d'un corps fixe et rigide qui connecte le monde extérieur au monde

46. Voir R. Descartes, Traité de l'homme, op. cit., vol. XI, pp. 177-178 : Ce sont les figures qui se tracent dans les esprits sur la superficie de la glande, «qui doivent être prises pour les idées, c'est-à-dire pour les formes ou images que l'âme raisonnable considérera immédiatement, lorsqu'étant unie à cette machine elle imaginera ou sentira quelque objet ». Il est à noter que cet usage corporaliste du terme idée disparaît dans la suite de l'œuvre. Le schème inspectionniste est également tacite dans la fameuse gravure de la Dioptrique où une tête d'homme examine par en dessous l'image des corps extérieurs peinte sur la rétine... Sur cette dualité des modèles cartésiens de la représentation sensible, voir l'article original de G. Hatfield, «Descartes'physiology and its relation to his psychology », dans J. Cottingham (ed.), The Cambridge Companion to Descartes, Cambridge, Cambridge University Press, 1992, pp. 335-370.

Revue philosophique, $\mathrm{n}^{\circ} 1 / 2014$, p. 47 à p. 72 
intérieur du sensorium. Il requiert le transport incessant de particules dans les milieux transparents, ainsi que la propagation de vibrations dans les fluides occupant les corps opaques. L'image cérébrale se trouve physiquement détachée de sa source et c'est donc dans un sens beaucoup plus fort que chez Descartes qu'elle est appelée à devenir l'objet propre et immédiat de la perception.

Il reste à tenter de joindre les fils du raisonnement métaphysique sur la présence de Dieu et ceux issus de la psycho-physiologie. Une chose peut être dite d'emblée : Newton ne mobilise pas à la légère un concept emprunté de sensorium dont il n'aurait pas lui-même mesuré toutes les implications, ajoutant après coup des tanquam pour réparer les plâtres d'une métaphore malencontreuse. Derrière le concept de sensorium, il y a une théorie de la perception très charpentée et très ancienne, dont Newton, il faut le noter, ne s'est jamais départi.

Une seconde remarque joue cependant contre un usage trop littéral de la métaphore : la perception humaine est un processus qui met en jeu des mouvements de particules matérielles et des vibrations, transmis par des organes externes puis internes, et au terme desquels une image se forme dans un espace restreint du cerveau. De l'ensemble de ce dispositif sensoriel, organes externes et internes, sensorium compris, on peut dire que Dieu précisément n'a point besoin. Dieu est partout et n'a pas besoin de voir les choses par leurs images. Si par aventure notre âme avait le pouvoir de sortir de notre corps pour aller directement s'imprégner des choses extérieures, nous serions pareillement dispensés d'organes, internes ou externes : nous percevrions les corps directement là où ils sont, comme Dieu lui-même le fait. La métaphore a donc quelque chose d'un paradoxe : Dieu, pour Newton, n'a pas d'organe, pas de sensorium, précisément parce qu'il perçoit directement les choses par sa présence immédiate.

Il reste que la métaphore est tout de même motivée par une analogie structurelle forte entre deux ordres de présence sensible : celle de Dieu aux corps placés dans l'espace, et celle du «principe sentant » humain aux images véhiculées dans le sensorium. Dans les deux cas, l'accent porte sur l'idée que l'interaction causale requiert la coprésence. Celle-ci peut prendre la forme d'une simple contiguïté, comme cela semble être le cas pour l'image cérébrale qui est représentée sur une simple «surface » au contact duquel se trouve notre principe sentant ; ou bien d'une compénétration, comme c'est nécessairement le cas pour Dieu, qui n'occupe pas seulement l'espace vide à l'extérieur des corps, mais est aussi intimement présent au corps, dans toute sa masse. Notons que Newton semble ne pas douter que 
cette compénétration soit métaphysiquement possible : l'impénétrabilité essentielle des corps ne s'y oppose pas, car Dieu n'est pas une substance corporelle. Dans le De Gravitatione, Newton envisageait même que l'impénétrabilité des corps les uns à l'égard des autres ne soit précisément que la manifestation de la présence de Dieu, un pouvoir communiqué librement à certaines portions d'espace vide au moment de la Création. Quoi qu'il en soit, la compénétration du corps et de l'esprit n'est pas hylémorphique : l'esprit habite l'espace corporel du sensorium, il en ressent les modifications, mais il n'en dépend point et n'en est pas la forme. Les deux substances ne sont pas réellement unies, elles restent indépendantes. Et c'est sans doute cette indépendance qui permet à la conscience perceptive de ne pas s'identifier aux images qu'elle perçoit, de les tenir « consciemment » à distance ${ }^{47}$.

Il reste à l'évidence un certain nombre de problèmes irrésolus. Nous avons relevé que l'application élargie de la métaphore du sensorium, qui permet à Newton d'expliquer dans le même mouvement la présence sensible de Dieu et sa présence active ou motrice, ne va pas de soi. En particulier, le mouvement volontaire participe à un processus d'identification et d'appropriation du corps propre, qui ne s'applique pas aux images perceptives. On peut même se demander s'il requiert au même titre que la perception la présence d'images cérébrales. Un autre problème concerne le type d'extension spatiale qu'il faut reconnaître à l'esprit humain ou divin. On pourrait penser que le principe même de coprésence qui est à la source de la métaphore du sensorium aurait dû conduire Newton à défendre une position proche de celle de More, affirmant que l'esprit est une substance étendue, diffusée dans l'espace quoiqu'indivisible, comme l'espace lui-même dont on ne peut séparer les parties. Avoir une idée ou une image sensorielle d'extension, c'est saisir simultanément les parties de cette étendue, et partant c'est avoir un esprit capable de se rendre coextensif aux images cérébrales pour en recevoir l'empreinte. On

47. On peut se demander si Newton, qui utilise au sujet des images cérébrales le terme latin de species, n'a pas encore, en dépit de son inscription dans la théorie moderne de la vision, conservé quelque réminiscence de la vieille doctrine scolastique de la présence « intentionnelle » des espèces. L'espèce ou l'image sensible, telle que pouvaient la décrire Suarez ou Rubio, possède un mode de présence spécial : elle n'existe pas substantiellement, indépendamment du corps extérieur dont elle est pour ainsi dire la présentation ubiquitaire ; elle n'est donc pas pleinement inhérente au sujet matériel (l'organe des sens) qui l'instancie, dans l'organe c'est un accident fantomatique qui ne pourvoit le sujet où il existe d'aucune capacité à produire des changements naturels. Voir A. Simmons, « Explaining Sense Perception: a Scholastic Challenge », Philosophical Studies,1994, 73, pp. 257-275.

Revue philosophique, $\mathrm{n}^{\circ}$ 1/2014, p. 47 à p. 72 
peut même dire que c'est en affirmant cette " coextensivité » de l'esprit et des images qu'on évite in fine de succomber au paradoxe de l'homoncule. Si en effet l'âme devait inspecter les images depuis un point inétendu qui serait à l'extérieur de ces images, le bénéfice de l'explication causale serait perdu : la perception se ferait de nouveau à distance, inintelligiblement.

Si Clarke paraît avoir tiré la conséquence ontologique naturelle (l'esprit est un être étendu, mais comme l'espace lui-même, il reste un et indivisible et ne peut être affecté par les corps $)^{48}$, Newton pour sa part résista assez fortement à cette implication. En témoignent les précisions adressées à Des Maizeaux, déjà relevés, ou encore certains manuscrits tardifs, dans lesquels il dit que ce n'est que par métaphore, ou par trope, que Dieu peut être nommé, comme il l'est dans la Kabbale, makom, le lieu ${ }^{49}$. Dans un texte très tardif, un ajout du Scholium Generale qui paraît dans la troisième édition des Principia de 1726, il propose de penser une omniprésence sans extensivité, sur un modèle fortement inspiré des thèses scolastiques " holenméristes » récusées par More :

Il y a des parties successives dans la durée et des parties co-existantes dans l'espace ; il n'y a rien de semblable dans ce qui constitue la personne de l'homme ou dans son principe pensant ; et bien moins y en aura-t-il dans la substance pensante de Dieu. Tout homme, en tant qu'il est un être sentant, est un seul et même homme pendant toute sa vie et dans tous les divers organes de ses sens. Ainsi Dieu est un seul et même Dieu partout et toujours ${ }^{50}$.

48. Clarke (Second Reply, 10 janv. 1716 ; éd. Robinet p. 48) expliquait à Leibniz que l'indivisibilité de l'âme ne prouve pas qu'elle soit réduite à un point, car l'espace qu'il soit fini ou infini est lui-même quelque chose d'indivisible imaginer qu'on puisse en séparer les parties c'est imaginer qu'on les meut hors d'elles-mêmes.

49. "When the Hebrews called God Makom place, the place in which we live, move and have our being \& yet did not mean that space is God in a litteral sense for they used to speak of God by figures and allusions and put space for his omnipresence by a figure » (Ms Cambridge, Portsmouth Collection Add 3965 fol. 290, cité in Copenhaver, "Jewish Theologies of Space in the Scientific Revolution ", art. cit. note 16).

50. Voir Scholium Generale (Philosophiae Naturalis Principia Mathematica, $3^{\text {rd }}$ ed., Londres 1726, p. *) : " Partes dantur successivae in duratione, coexistentes in spatio, neutrae in persona hominis seu principio ejus cogitante; \& multo minus in substantia cogitante dei. Omnis homo, quatenus res sentiens, est unus \& idem homo durante vita sua in omnibus \& singulis sensuum organis. Deus est unus \& idem deus semper et ubique. " ("Il y a des parties successives dans la durée, et des parties co-existantes dans l'espace ; il n'y a rien de semblable dans ce qui constitue la personne de l'homme ou dans son principe pensant; et bien moins y en aura-t-il dans la substance pensante de Dieu. Tout homme, en tant qu'il est un Être sentant, est un seul et même homme pendant toute sa vie et dans tous les divers organes de ses sens. Ainsi Dieu est un seul et même Dieu partout et toujours. Il est présent partout, non seulement virtuellement, mais substantiellement, 
Dieu serait, ainsi, non pas étendu ou diffusé dans l'espace, ni substantiellement identique à celui-ci, mais totum in qualibet parte ; présent tout entier en chacune des parties de l'univers - tout comme l'homme est toujours et partout la même personne, quelles que soient les modifications qui adviennent à son corps ou à son esprit, qu'il soit en train de voir, d'entendre ou de penser. Dieu, écrit encore Newton, est « tout œil, tout oreille, tout cerveau, tout bras, tout pouvoir de percevoir, d'entendre ou d'agir ». Il est toujours, tout entier lui-même, en chacun de ses actes, la même personne indivisible ${ }^{51}$.

Il semble donc que la toute dernière métaphysique newtonienne présente une tension entre deux modèles humains concurrents et potentiellement contradictoires pour penser l'omniprésence divine. Dans le modèle du sensorium, convoqué dans les queries pour concevoir l'activité et la souveraineté de Dieu, on s'appuie sur l'idée que la substance spirituelle est littéralement étendue dans un espace privilégié, un siège de l'âme, et l'on affirme cette coextensivité de l'esprit et du corps comme la condition de l'activité perceptive et motrice. Dans le modèle de la personne humaine, convoqué dans le Scholium pour concevoir l'unité et l'indivisibilité de Dieu, on s'appuie sur l'unité substantielle et indivisible d'un même sujet hylémorphique, qu'on ne peut identifier à l'extension d'aucune partie ou d'aucun organe particulier, parce qu'elle est tout entière présente en chacun. Les deux modèles sont clairement distincts ${ }^{52}$, et Newton qui ne mentionne plus le sensorium dei dans le Scholium Generale, n'a manifestement pas cherché à les rendre compatibles.

Philippe НамоU Institut de Recherches Philosophiques (EA 373), Université Paris Ouest Nanterre phamou@u-paris10.fr

car on ne peut agir où l'on n'est pas. "; Newton, Principes mathématiques, op. cit., pp. 176-177). Pour la chronologie des révisions du Scholium entre 1713 et 1726, voir A. R. Hall \& M. Boas Hall, Unpublished Scientific Papers of Isaac Newton, Cambridge, Cambridge University Press, 1978, pp. 348 sqq.

51. L'identité de la persona divine est ici pensée de manière traditionnelle comme l'identité substantielle d'une même hypostase - et à cet égard l'influence sur ce texte du chapitre sur l'identité personnelle de l'Essai sur l'entendement humain de Locke, qui met l'accent sur le critère de conscience, paraît peu vraisemblable.

52. Comme le montre sur le cas cartésien M. Rozemond, «Descartes, MindBody Union and Holenmerism », Philosophical Topics, 2003, 31, pp. 343-367. 